\begin{tabular}{|c|c|}
\hline & $\begin{array}{l}\text { International Journal of Trend in Scientific } \\
\text { Research and Development (IJTSRD) }\end{array}$ \\
\hline 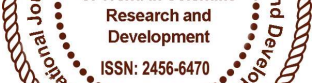 & International Open Access Journal \\
\hline 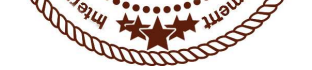 & ISSN No: 2456 - 6470 | www.ijtsrd.com | Volume - 2 | Issue - 2 \\
\hline
\end{tabular}

\title{
GRS - Gesture based Recognition System for Indian Sign Language Recognition System for Deaf and Dumb People
}

\author{
D. Anbarasan, R. Aravind, K. Alice \\ Department of Computer Science and Engineering, \\ G.K.M. College of Engineering and Technology, Chennai, India
}

\section{ABSTRACT}

Recognition languages are developed for the better communication of the challenged people. The recognition signs include the combination of various with hand gestures, movement, arms and facial expressions to convey the words thought. The languages used in sign are rich and complex as equal as to languages that are spoken. As the technological world is growing rapidly, the sign languages for human are made to recognised by systems in order to improve the accuracy and the multiply the various sign languages with newer forms. In order to improve the accuracy in detecting the input sign, a model has been proposed. The proposed model consists of three phases a training phase, a testing phase and a storage output phase. A gesture is extracted from the given input picture. The extracted image is processed to remove the background noise data with the help of threshold pixel image value. After the removal of noise from the image and the filtered image to trained model is tested with a user input and then the detection accuracy is measured. A total of 50 sign gestures were loaded into the training model. The trained model accuracy is measured and then the output is extracted in the form of the mentioned language symbol. The detection mechanism of the proposed model is compared with the other detection methods such as Hidden Markov Model(HMM), Convolutional Neural Networks(CNN) and Support Vector Machine(SVM). The classification is done by means of a Support Vector Machine(SVM) which classifies at a higher accuracy. The accuracy obtained was 99 percent in comparison with the other detection methods.

Keywords: Gesture recognition, pattern classification, Image extraction, Hidden Markov Model (HMM), Convolutional Neural Network (CNN), Support Vector Machine (SVM)

\section{INTRODUCTION}

The natural way of exchanging information among the deaf and dumb is sign language. In order to overcome the difficulties faced by deaf and dumb people when they communicate with normal people sign recognition language was invented. There are three types of sign languages Indian Sign Language, British Sign Language and American Sign Language. Only 5\% of Indian deaf and dumb people get education in order to increase educating the deaf and dumb people sign languages are used. Indian Sign Language has its own specific syntax, grammar, alphabets, words and numerals. The gestures are made with the help of combination of fingers, hand, wrist movements and elbow movements. There are two types of aspects one is by positioning the finger and another one is by changing the orientations. The sign language needed to be well structured for a higher level of understanding. The sign language is process involves image acquisition with image classification. Facial expressions are ignored as they are ineffective of the substantial expressions in it. For improving the accuracy and a new method has been proposed. The 
method includes the combination of the various data expressions involving sign nature. The method detected with a $99 \%$ accuracy.

\section{RELATED WORKS}

An Sandeep kaul and manider singh[2015] designed a model with SigML and HamNoSys. The JA SIGML URL App was developed inorder to verify the HamNo input. The inputs were in the form of HamNoSys notations. The accuracy of the animated signs is tested with the help of "Indian Sign Language Dictionary". The main disadvantage of the model no concurrent words are recognized. A novel technique focusing on transformation content of sign language images by Nalini Yadav et. al [2015]. The goal of the proposed classification technique provided a faster retrieval with higher accuracy. Kumad Tripathi et. al proposed a system by combining Orientation Histogram and Principal Component Analysis(PCA) which reduced the dimension features after extraction. Combined the correlation and Euclidean distance which gave an higher accuracy. [2015]. Asha Thalange and Dr. S. K. Dixit.[2016] Proposed two new feature extraction techniques naming them as Combined Orientation Histogram and Statistical(COHST) features and Wavelet Features for recognition of static signs of numbers . It was implemented using Neural networks. The proposed system was with an accuracy of $98.12 \%$ of recognition. Surbhi rahat and ujwal gandat [2017] proposed a vision based method inducing processing, feature extraction, segmentation and classification. The vision based method enhances with various approaches that converts the text into voice module and classifies it. This method not implemented the inducing of sentence conversation.

Neela harish and Poonguzhali [2015] proposed a system which included flex sensors and accelerometer fixed to the fingers and wrist. These sensors are fitted with the help of glove. The text were converted with the help of the electric signals produced interms of voltage and the voice output were produced and sent to the voice module producing appropriate words. Prof. Prasanth G et. al [2015] designed a system for processing video to speech will include frame formation from videos and region of interst with language knowledge base using correlational based approach than the audio conversion. The mapping of text and relevant animated gestures from the database are implemented. It was not designed for American Sign Language. A smart glove technique using Intel Galileo Gen2 IOT kit for real time gesture recognition by Abinandhan Das et. al/. The device included both sign language recognition and gesture recognition system[2016].

\section{PROPOSED SYSTEM}

The proposed system developed to efficiently detect the gestures recognized at a high accuracy. The Gesture Recognition System (GRS) consists of three phases through which the output for the input gesture is observed. The three phases involve mainly a training phase, a dataset training storage phase and a testing phase.

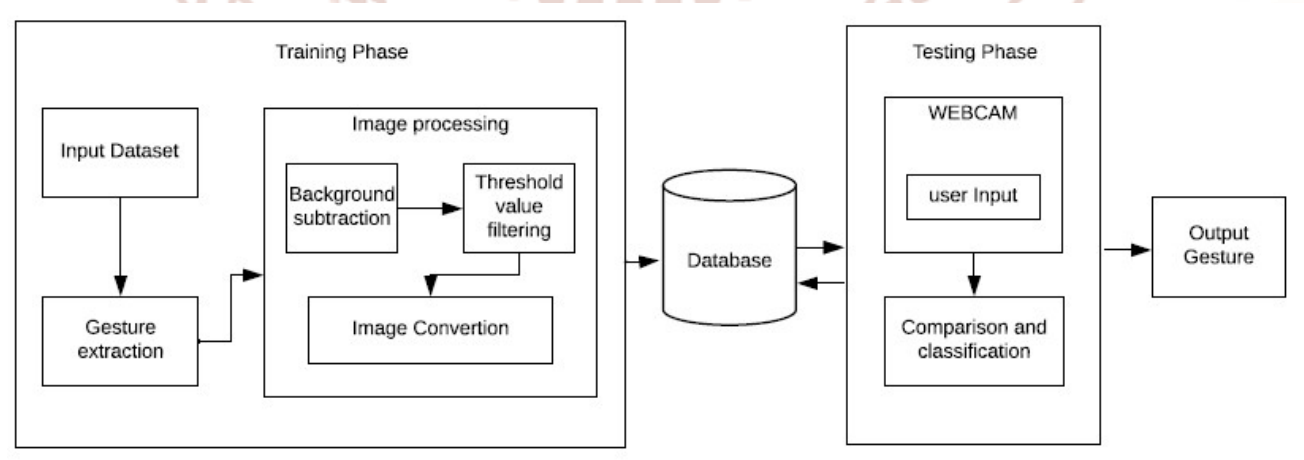

Fig.1 Architecture of GRS

\section{A. Training phase}

The entire document should be in Times New Roman or Times font. Type 3 fonts must not be used. Other font types may be used if needed for special purposes.
The training phase is primarily provided with an input symbol recognition dataset. The dataset involves the various symbols that have different pictures that denote the various gestures for English alphabets and 
numbers. The dataset also involves various background noises i.e. data is not preprocessed.

\section{i. $\quad$ Gesture extraction}

The input symbols involve various background data which may be considered as noise or outliers. Then with the help of the color intensity variation and edge detection, gestures are recognized. The Gestures are recognized with the edge detection. Further, the image needs preprocessing for the accurate gesture detection.

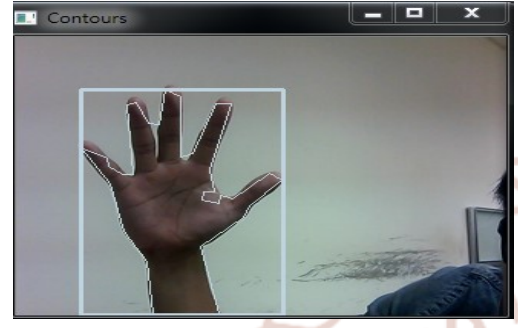

Fig.2 Extraction of Gesture

\section{ii. Image Processing}

The image with a detected edge is then moved for edge filtering. An image filter for background subtraction is implemented. The background image is omitted with the removal of the components outside the edge detected. The edge contains some of the input data that may not be recognized for filtering. A threshold value is respect to the intensity of the data that is processed. The portion of the image that matches the intensity value is retained and others are filtered. Finally, an image observed is converted into the input format which is supportable to be given as the input dataset for the testing phase.
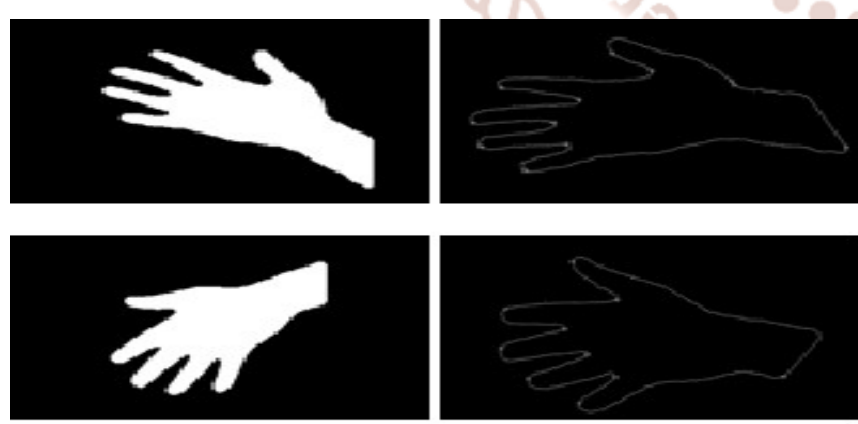

Fig.3 Processing of image

\section{B. Storage Phase}

The input images extracted with a gesture symbols is stored onto the database storage. A dynamic data storage database is used here where the new gestures can be feed as the input. The gestures stored onto the database are moved onto the testing phase to test the accuracy of the model.

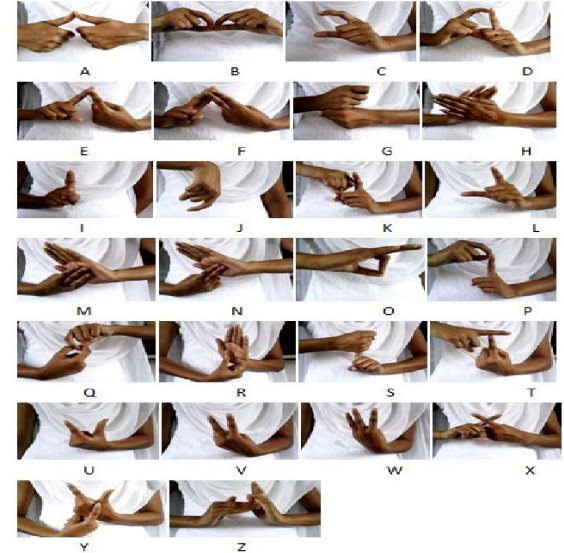

Fig.4 Different signs of ISL

\section{Testing Phase}

The testing phase implemented with the data from the user. The user data is given by a web camera. The web camera provides the gesture of the input hand sign. The edges of the fingers are detected in the system. The points of the edge are recognized. The background images rather than the edges detected are removed by the model. The gesture recognized is the classified with given input datasets and returns the maximum probable gesture. It returns the various gestures that result above the threshold value. The comparison module compares the classified gestures with the input pattern. Enhance the recognized pattern with nearest sign image. The image that matches the nearest value is then returned to the database. The database data that matches the nearest value for the sign is returned as the output with a high accuracy.

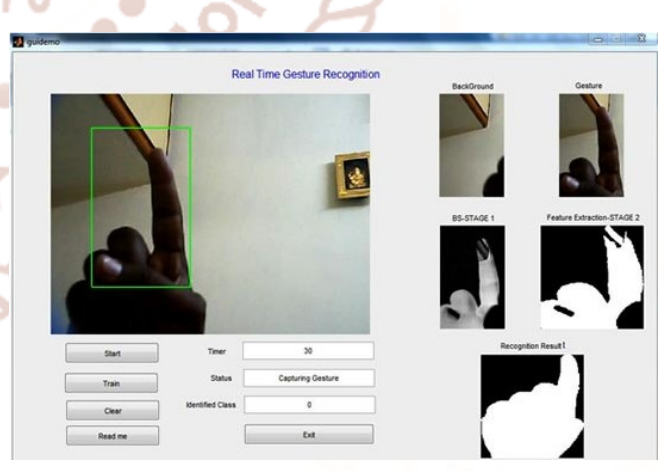

Fig.5 Gesture testing of ISL

\section{Experimental evaluation}

The experimental setup is done by means of Java IDE with python environment. The four detection methods are built in python code. The simulation environment is build for 1, 50, 100 and 500 datasets. Two efficient metrics were observed in the environment. Firstly, the time taken to detect the sign that has been recognized. The GRS model tended to detect the sign feed within 
the shortest amount of time as given table 1.1 . Secondly, the detection accuracy is measured with the help of the precision and recall metrics which were observed that all the signs given into the system were observed accurately.

\section{TABLE I}

GESTURE DETECTION TIME FOR DIFFERENT METHODS

\begin{tabular}{|c|c|c|c|c|}
\hline $\begin{array}{l}\text { Detection } \\
\text { Methods }\end{array}$ & $n=1$ & $n=50$ & $n=100$ & $n=500$ \\
\hline HМM & 0.0924 & 0.0864 & 0.0864 & 0.0859 \\
\hline CNN & 0.0726 & 0.0677 & 0.0669 & 0.0665 \\
\hline SVM & 0.0735 & 0.0684 & 0.0677 & 0.0674 \\
\hline GRS & 0.07 & 0.0673 & 0.0667 & 0.0665 \\
\hline
\end{tabular}

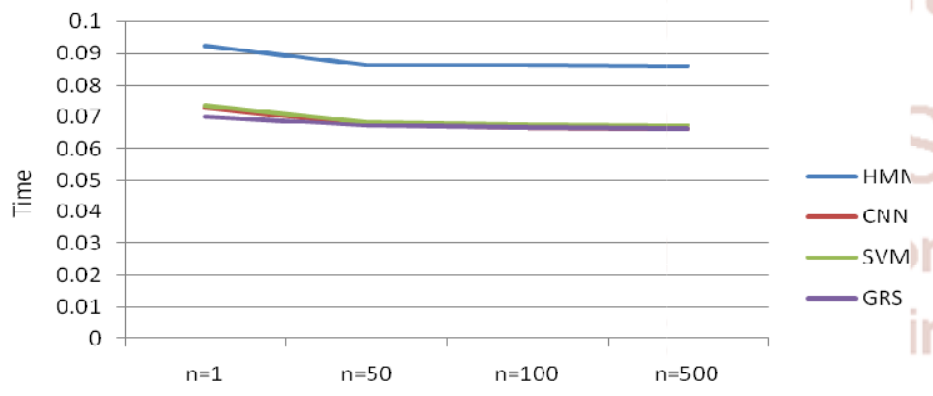

Number of datasets

Fig.6 Time taken by GRS for output

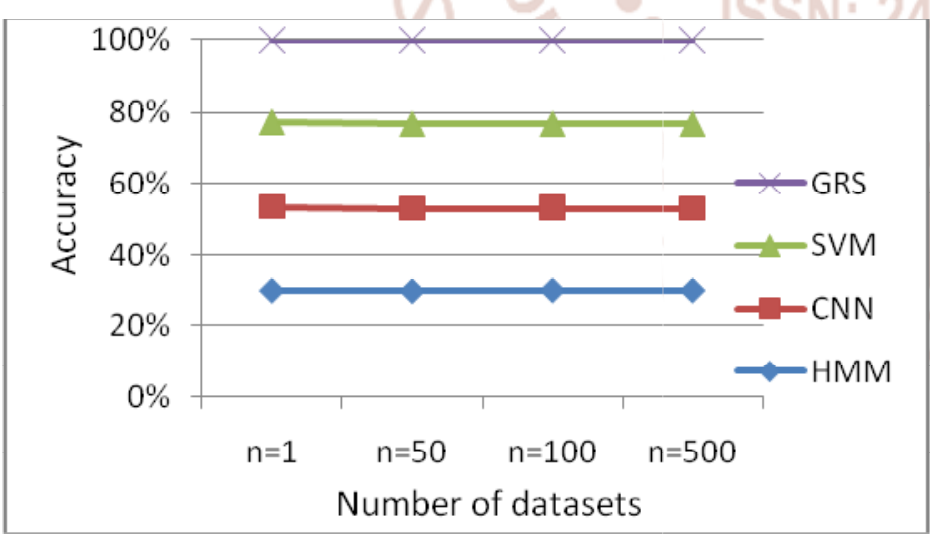

Fig. 7 Accuracy of GRS

\section{E. Conclusions}

The Gestures Recognition System is developed through this model. The GRS model recognizes the input signal with a complete accuracy. The Combination of the phases induced the system with larger symbol detection mechanisms. Comparison of recognition techniques such as HMM, CNN and SVM, the GRS technique concluded to have an higher classification and recognition of various symbols in ISL. The model classified 500 symbols. The GRS will be a boon to the challenged people and to gain higher standards in education. The future work is to develop a mobile application for the sign recognition system.

\section{REFERENCES}

1) Rojasara, D. and Chitaliya, N., 2014. Real Time Visual Recognition of Indian Sign Language using Wavelet Transform and Principle Component Analysis. International Journal of Soft Computing and Engineering (IJSCE), 4(3), pp.17-20.

2) Thalange, A. and Dixit, S.K., 2016. COHST and Wavelet Features Based Static ASL Numbers Recognition. Procedia Computer Science, 92, pp.455-460.

3) Tripathi, K. and Nandi, N.B.G., 2015. Continuous indian sign language gesture recognition and sentence formation. Procedia Computer Science, 54, pp.523-531.

4) Rajaganapathy, S., Aravind, B., Keerthana, B. and Sivagami, M., 2015. Conversation of sign language to speech with human gestures. Procedia Computer Science, 50, pp.10-15.

5) Suharjito, S., Sign Language Recognition Application Systems for Deaf-Mute People: A Review Based on Input-Process-Output.

6) Anderson, R., Wiryana, F., Ariesta, M.C. and Kusuma, G.P., 2017. Sign Language Recognition Application Systems for Deaf-Mute People: A Review Based on Input-Process-Output. Procedia Computer Science, 116, pp.441-448.

7) Das, S.P., Talukdar, A.K. and Sarma, K.K., 2015. Sign Language Recognition using Facial Expression. Procedia Computer Science, 58, pp.210-216.

8) Chavan, P., Ghorpade, T. and Padiya, P., 2016, March. Indian sign language to forecast text using leap motion sensor and RF classifier. In Colossal Data Analysis and Networking (CDAN), Symposium on (pp. 1-5). IEEE.

9) Gupta, B., Shukla, P. and Mittal, A., 2016, January. K-nearest correlated neighbor classification for Indian sign language gesture recognition using feature fusion. In Computer Communication and Informatics (ICCCI), 2016 International Conference on (pp. 1-5). IEEE. 
10) Kim, S.Y., Han, H.G., Kim, J.W., Lee, S. and Kim, T.W., 2017. A Hand Gesture Recognition Sensor Using Reflected Impulses. IEEE Sensors Journal, 17(10), pp.2975-2976.

11) Li, T.H.S., Kao, M.C. and Kuo, P.H., 2016. Recognition System for Home-Service-Related Sign Language Using Entropy-Based \$ K \$Means Algorithm and ABC-Based HMM. IEEE Transactions on Systems, Man, and Cybernetics: Systems, 46(1), pp.150-162.

12) Wu, J., Sun, L. and Jafari, R., 2016. A wearable system for recognizing American sign language in real-time using IMU and surface EMG sensors. IEEE journal of biomedical and health informatics, 20(5), pp.1281-1290.

13) Elmahgiubi, M., Ennajar, M., Drawil, N. and Elbuni, M.S., 2015, June. Sign language translator and gesture recognition. In Computer \& Information Technology (GSCIT), 2015 Global Summit on (pp. 1-6). IEEE. 